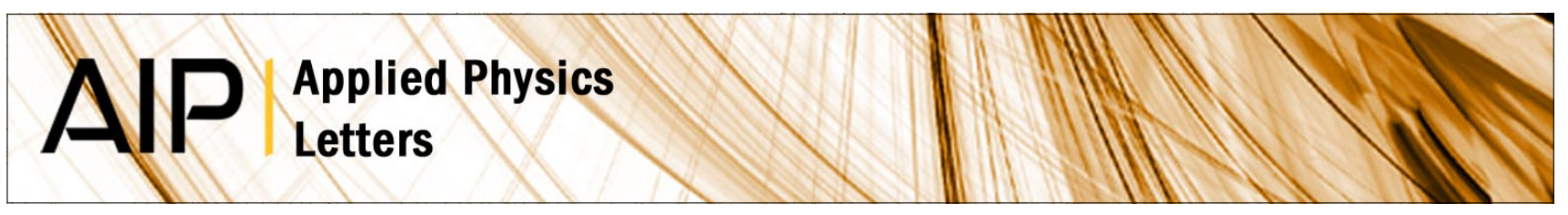

\title{
Control of chaos in a $\mathrm{CO} 2$ laser
}

J. M. Perez, J. Steinshnider, R. E. Stallcup, and A. F. Aviles

Citation: Appl. Phys. Lett. 65, 1216 (1994); doi: 10.1063/1.112075

View online: http://dx.doi.org/10.1063/1.112075

View Table of Contents: http://apl.aip.org/resource/1/APPLAB/v65/i10

Published by the American Institute of Physics.

\section{Related Articles}

High coherent bi-chromatic laser with gigahertz splitting produced by the high diffraction orders of acousto-optic modulator used for coherent population trapping experiments

Rev. Sci. Instrum. 82, 123104 (2011)

Self-mixing interferometry in vertical-cavity surface-emitting lasers for nanomechanical cantilever sensing Appl. Phys. Lett. 94, 091103 (2009)

Field fluctuations and spectral line shape in semiconductor lasers subjected to optical feedback J. Appl. Phys. 95, 7573 (2004)

Supermode-noise suppression using a nonlinear Fabry-Pérot filter in a harmonically mode-locked fiber ring laser Appl. Phys. Lett. 81, 4520 (2002)

Modulation doped InGaAsP quantum well laser emitting at $1.55 \mu \mathrm{m}$

J. Appl. Phys. 90, 38 (2001)

\section{Additional information on Appl. Phys. Lett.}

Journal Homepage: http://apl.aip.org/

Journal Information: http://apl.aip.org/about/about_the_journal

Top downloads: http://apl.aip.org/features/most_downloaded

Information for Authors: http://apl.aip.org/authors

\section{ADVERTISEMENT}

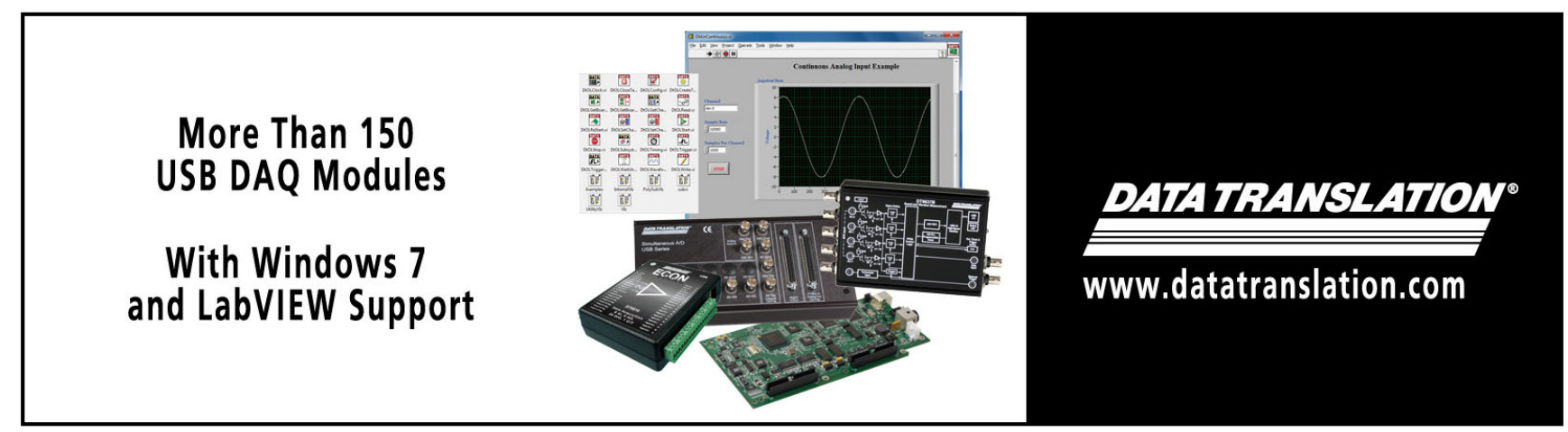




\section{Control of chaos in a $\mathrm{CO}_{2}$ laser}

J. M. Perez, J. Steinshnider, R. E. Stallcup, and A. F. Aviles

Department of Physics, University of North Texas, Denton, Texas 76203-5368

(Received 3 March 1994; accepted for publication 23 June 1994)

We report the experimental control of chaos in an optically modulated $\mathrm{CO}_{2}$ laser. The $\mathrm{CO}_{2}$ laser was driven into chaos by injecting a feedback beam modulated by an electro-optical modulator. Control of chaos was achieved using a modified proportional feedback technique in which the control pulses were delayed by approximately one relaxation period. Using this technique, it was possible to control unstable periodic orbits up to period 6 .

Recently, Ott, Grebogi, and Yorke (OGY) ${ }^{1}$ showed that it was possible to control chaos in nonlinear systems by applying small time-dependent perturbations to a system parameter. There is currently considerable interest in applying this technique to experimental systems. ${ }^{2,3}$ Using techniques based on the OGY method, experimental control of chaos in a Nd-doped yttrium aluminum garnet (YAG) laser $^{4}$ and a Nd-doped optical fiber laser ${ }^{5}$ have recently been reported. In these cases, the control pulses were applied to the pump of the laser. In this paper, we report, for the first time, experimental control of chaos in an optically modulated $\mathrm{CO}_{2}$ laser. Control in this case is achieved by applying the control pulses to the $Q$ of the laser instead of the pump. Using this technique, control of unstable orbits up to period 6 could easily be achieved.

$\mathrm{CO}_{2}$ lasers have been experimentally shown to become chaotic as a result of $Q$ switching, ${ }^{6}$ delayed feedback, ${ }^{7}$ and coupling with other $\mathrm{CO}_{2}$ lasers. ${ }^{8}$ Our experimental setup is shown in Fig. 1. The $\mathrm{CO}_{2}$ laser is a two meter long, gas flow, longitudinally excited laser with a dc excitation voltage of 20 $\mathrm{kV}$ and current of $10 \mathrm{~mA}$. The cavity is stabilized with Invar rods, and a diffraction grating is used to select a single mode at $10.6 \mu \mathrm{m}$. Brewster windows are used to linearly polarize the lascr beam which allows intensity modulation of the beam using a CdTe electro-optical modulator (EOM). The EOM is located outside the laser cavity, as shown in Fig. 1. The EOM modulates the polarization of the output laser beam, and the beam is then reflected back to the laser cavity using a mirror mounted on a piezoelectric transducer. Using the piezoelectric transducer, the modes of the external cavity can be adjusted to be resonant with the modes of the laser cavity.

The intensity of the laser beam is measured using liquid nitrogen cooled $\mathrm{HgCdTe}$ photodiode detector and amplifier with a bandwidth of $1 \mathrm{MHz}$. The detector was positioned to measure the intensity of the laser beam reflected from the diffraction grating, as shown in Fig. 1. Laser beam intensity vs time, $I(t)$, and the phase portraits of $I(t)$ vs $I(t+\tau)$, where $\tau$ is a delay time, were obtained on an oscilloscope using a transmission delay line with a fixed delay time of approximately $7.5 \mu \mathrm{s}$. Frequency spectra of $I(t)$ were obtained using a IIewlett Packard 3585A $20 \mathrm{~Hz}-40 \mathrm{MHz}$ spectrum analyzer. $I(t)$ and $I(t+\tau)$ were also digitized using a two channel, $8 \mathrm{bit}, 10 \mathrm{MHz}$ digitizer.

The EOM was driven between $50-84 \mathrm{kHz}$ near the re- laxation oscillations of the $\mathrm{CO}_{2}$ laser. The driving voltage was approximately $3 \mathrm{kv}$ peak to peak and had an offset voltage of $1.5 \mathrm{kV}$. In this frequency region, chaos in $I(t)$ was observed after several period doubling bifurcations, as shown in Fig. 2(a).

Control of chaos was obtained by applying the control pulses to the EOM. Previous studies of control of chaos in a YAG laser and Nd-doped optical fiber laser applied the control pulses to the pump which was a laser diode. ${ }^{4,5}$ However, the $\mathrm{CO}_{2}$ laser is relatively insensitive to pump modulation. Attempts to experimentally control chaos in the $\mathrm{CO}_{2}$ laser by applying the control pulses to the pump were not successful. To control chaos in the $\mathrm{CO}_{2}$ laser, we used a modification of the proportional feedback technique described in Ref. 2 . In this technique, the system's dynamics are analyzed by a window comparator and a control pulse is fed back to the control parametcr. In our case, the laser beam pulses are analyzed by a window comparator and the control pulses are fed back to the EOM. A control pulse is triggered only if the laser beam pulse peak falls in the window. The magnitude of the control pulse is determined by the position of the laser beam pulse peak in the window. The window width and offset height are variable parameters. In addition, we used a variable delay of approximately one drive period between the control pulse and laser beam pulse. Using this delay, the control pulse could be applied to the EOM during the subsequent laser beam pulse. This delay was necessary because the control

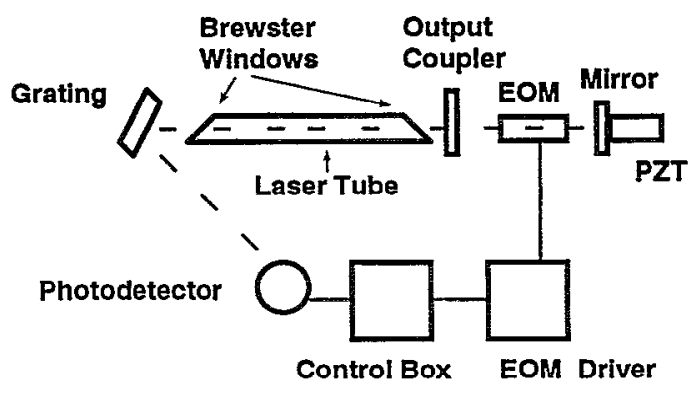

FIG. 1. $\mathrm{CO}_{2}$ laser system. The electro-optical modulator (EOM) is located outside the laser cavity. An external cavity is formed by the output coupler and mirror. The mirror reflects the output laser beam back into the laser cavity. 
(a)

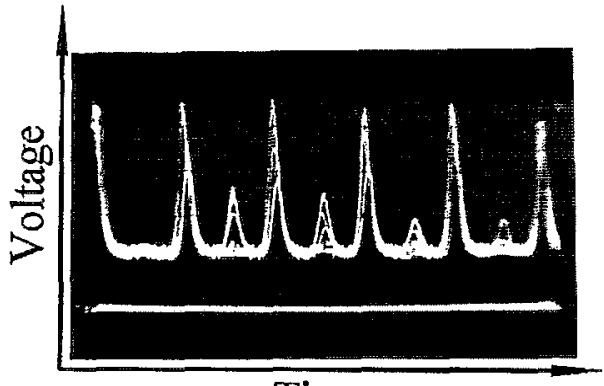

Time

(b)

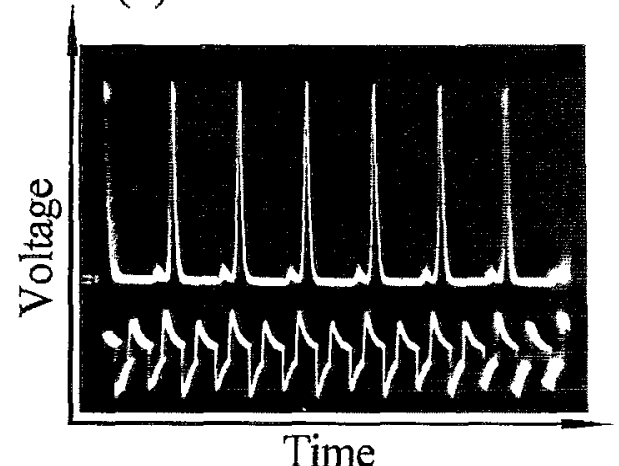

FIG. 2. (a) Chaotic time series of the $\mathrm{CO}_{2}$ laser system. Horizontal axis is time, with $200 \mu$ s full scale. Vertical axis is voltage in arbitrary units. (b) Top curve is the time series of the beam intensity of the controlled $\mathrm{CO}_{2}$ laser system. Bottom curve is the time series of the control pulses. Axes units are as in (a).

electronics were not fast enough to act on the laser beam pulse that triggered the control pulse.

In an experiment, the output of the photodetector was fed to the control electronics described above and the window width, offset height, and delay varied to achieve control. The control pulses were approximately $1 \%$ of the EOM driving voltage. Figure 2 (a) shows the chaotic time series, $I(t)$, for no control for a drive frequency of $50.2 \mathrm{kHz}$ and a laser current of $3.77 \mathrm{~mA}$. In Fig. 2(a), the top trace is $I(t)$ and the bottom trace is the control pulse signal. Using the control technique described above, the chaotic time series shown in Fig. 2(a) could be controlled to a period 2 orbit, as shown in Fig. 2(b). In Fig. 2(b), the top trace is $I(t)$ and the bottom trace is the control pulse signal. In the figure, the control pulse signal is greatly amplified for visibility. Figures 3(a) and 3(b) show $I(t)$ versus $I(t+\tau)$ for the time series shown in Figs. 2(a) and 2(b), respectively. Figures 4(a) and 4(b) show the frequency spectrum of the time series shown in Figs. 2(a) and 2(b), respectively. By changing the window width, offset height, and delay of the control electronics, we were able to control a period 6 orbit for the same laser system parameters as in Fig. 2(a), as shown in Fig. 5. In Fig. 5, the top oscilloscope trace is $I(t)$ and the bottom trace is the control pulse signal. The period of the controlled orbit is 6 counted in terms of the modulation period of the EOM. The tick marks above the figure denote the drive period. The (a)

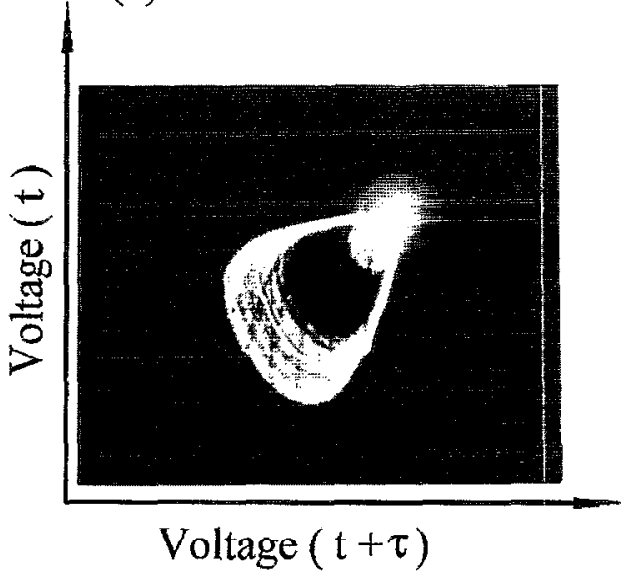

(b)

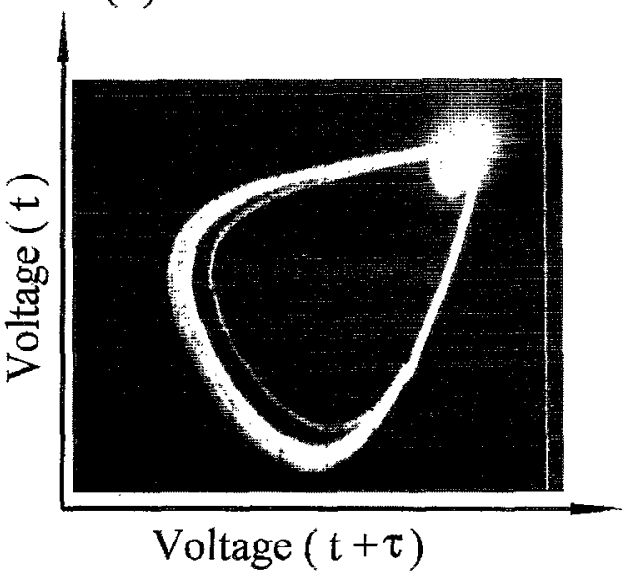

FIG. 3. (a) Phase portrait corresponding to the time series of Fig. 2(a). (b) Phaser portrait corresponding to the controlled time series of Fig. 2(b). Axes in (b) correspond to $\times 2$ magnification of (a). $\tau$ for both phase portraits is the delay time.

control pulses are being applied at $2 / 3$ the frequency of the modulation. Since the control pulses are only $1 \%$ of the drive amplitude, we believe these pulses are controlling unstable orbits instead of changing the underlying system dynamics. This is further substantiated by the fact that changing the amplitude of the EOM drive voltage uniformly by $1 \%$ did not result in any observable change in the chaotic attractor.

A preliminary model for the laser system is given by the differential equations ${ }^{9}$

$$
\begin{aligned}
& \frac{d I}{d t}=-k(1+\epsilon \cos \Omega t) I+\alpha I N, \\
& \frac{d N}{d t}=-\gamma_{\|}\left(N-N_{0}\right)-2 \alpha I N,
\end{aligned}
$$

where $I$ is the laser intensity, $k$ is the intensity decay constant, $N$ is the population inversion, $\alpha$ is the coupling coefficient for $I$ and $N, \eta_{\|}$is the population inversion decay constant, and $N_{0}$ is the pumping power. ${ }^{9}$ The reinjected beam in our system is modeled by the term $-k^{\prime} I \epsilon \cos \Omega t$, where $k^{\prime}$ is the intensity decay constant of the external cavity, which 
(a)

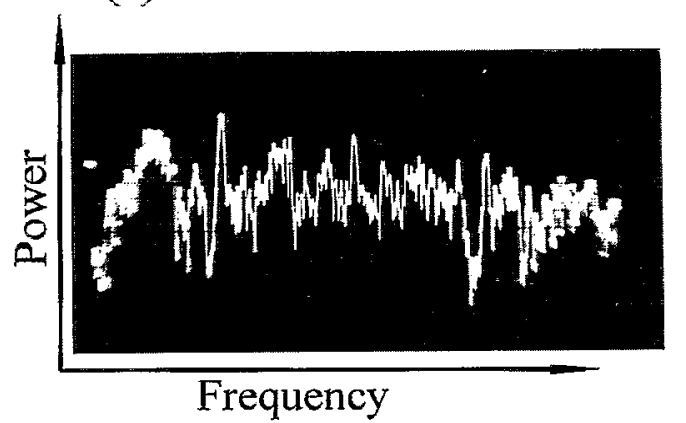

(b)

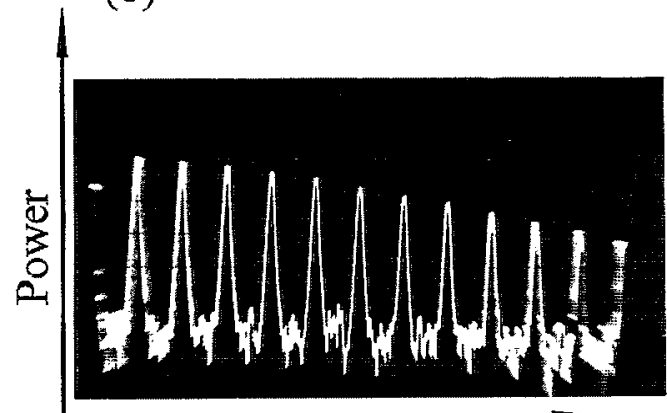

Frequency

FIG. 4. (a) Frequency spectrum corresponding to the time series of Fig. 2(a). Horizontal axis is frequency, with $200 \mathrm{kHz}$ full scale. Vertical axis is power in arbitrary units. (b) Frequency spectrum corresponding to the time series of Fig. 2(b). Axes are as in (a).

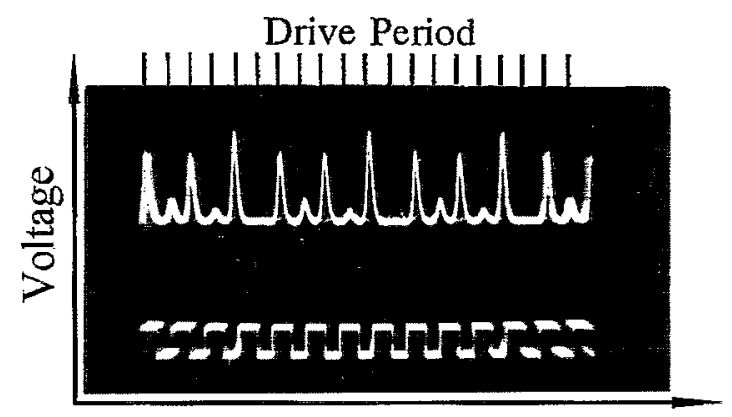

Time

FIG. 5. Time series of the controlled period 6 orbit. The period of the orbit is counted in terms of the modulation period of the EOM. The tick marks above the figure denote the modulation period. Axes units are as in Fig. 2(a).

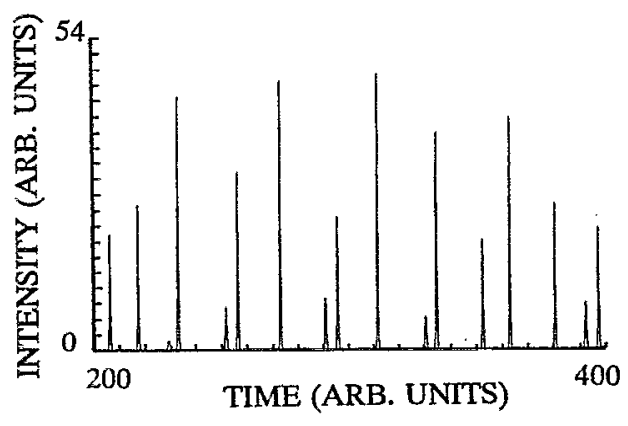

FIG. 6. Simulated chaotic time series of the laser beam intensity for our $\mathrm{CO}_{2}$ laser system.

we assume has a magnitude on the order of $k$, and $I \epsilon \cos \Omega \iota$ represents the modulation of the EOM where $\epsilon$ is the modulation depth and $\Omega$ is the driving frequency of the modulation. This model is preliminary and does not include the time delay of the light being fed back to the cavity nor the laser field phase. A more accurate model will be published later. We solved equations (1) and (2) numerically using a modulation frequency of $50.2 \mathrm{kHz}$ near the experimental modulation frequency used in Fig. 2(a). Chaos was easily achieved for small modulation depths of $1-5 \%$, as shown in Fig. 6 .

In summary, we have controlled chan in an optically modulated $\mathrm{CO}_{2}$ laser using a modified proportional feedback technique. The control pulses were applied to the laser cavity losses. We are currently applying this technique to the stabilization of systems of coupled $\mathrm{CO}_{2}$ lasers.

${ }^{1}$ E. Ott, C. Grebogi, and J. A. Yorke, Phys.Rev. Lett. 64, 1196 (1990).

${ }^{2}$ E. R. Hunt, Phys. Rev. Lett. 67, 1953 (1991).

${ }^{3}$ J. Singer, Y-Z. Wang, and H. H. Bau, Phys. Rev. Lett. 66, 1123 (1991).

${ }^{4}$ R. Roy, T. W. Murphy, Jr., T. D. Maier, Z. Gills, and E. R. Hunt, Phys. Rev. Lett. 68, 1259 (1992).

${ }^{5}$ S. Bielawski, D. Derozier, and Pierre Glorieux, Phys. Rev. A 47, 2492 (1993).

${ }^{6}$ F. T. Arecchi, R. Meucci, G. P. Puccioni, and J. R. Tredicce, Phys. Rev. Lett. 49, 1217 (1982).

${ }^{7}$ F. T. Arecchi, G. Giacomelli, A. Lapucci, and R. Meucci, Phys. Rev. A 49, 4997 (1991).

${ }^{8}$ V. V. Likhanskii and A. P. Napartovich, Sov. Phys. Usp. 33, 228 (1990).

${ }^{9}$ See, for example, 0 . Svelto, Principles of Lasers (Plenum, New York, 1989). 